\title{
Short-Term Corneal Response to Corneal Refractive Therapy for Different Refractive Targets
}

\author{
Cesar Villa Collar, OD, MSc, FAAO, * José Manuel González-Méijome, OD, PhD, FIACLE, $†$ \\ Antonio Queirós, $\mathrm{OD}$, $†$ and Jorge Jorge, OD, PhD†
}

\begin{abstract}
Purpose: The goal of the present study was to investigate the shortterm variations in corneal topography within the first 3 hours of lens wear under open eye conditions, which can be considered an off-label condition in current orthokeratology treatment, and the recovery of the effect during an additional 3-hour period after lens removal.

Methods: Fourteen volunteers were fitted with Paragon CRT reverse geometry rigid gas permeable contact lenses simulating 2 different refractive targets of -2.00 and -4.00 diopters of myopia correction in the right and left eyes in random order. After the lenses were placed in both eyes, subsequent measurements of corneal topography were obtained at 30,60 , and 180 minutes; the same measurements were also taken at 30,60, and 180 minutes after lens removal.
\end{abstract}

Results: Significant flattening of the cornea was evident after 30 minutes of lens wear. Overall, the -4.00 group changes progressed more rapidly than in the -2.00 group, and they also took more time to recover after lens removal. However, for most of the parameters, differences between -2.00 and -4.00 treatments are evident only after 60 minutes of lens wear.

Conclusions: Under open eye conditions, the first 30 minutes of corneal refractive therapy is determinant to establish the myopic orthokeratologic effect and changes are almost identical irrespective of the refractive target. After this point, changes are different depending on the refractive target. The recovery was almost as fast as the onset of the orthokeratologic effect, demonstrating that the cornea deforms quickly beneath these lenses and does recover safely and quickly once the lenses are removed.

Key Words: corneal refractive therapy, corneal topography, orthokeratology, short-term effect, myopia correction, refractive target

(Cornea 2008;00:000-000)

Received for publication February 3, 2008; revision received July 15, 2008; accepted August 11, 2008.

From the*Clínica Oftalmologica Novovision S.L., Madrid, Spain; and $\dagger$ Department of Physics (Optometry), School of Sciences, University of Minho, Braga, Portugal.

Supported in part by a grant from the Science and Technology Foundation, Ministry of Science and Superior Education, and European Social Fund under contract 8281/2002 granted to J.M.G.-M.

Reprints: José Manuel González-Méijome, OD, PhD, FIACLE, Department of Physics (Optometry), School of Sciences, Campus de Gualtar, University of Minho, 4710-057 Braga, Portugal (e-mail: jgmeijome@fisica.uminho. pt).

Copyright (c) 2008 by Lippincott Williams \& Wilkins n recent years, our knowledge and understanding of contact lens corneal reshaping, corneal refractive therapy (CRT), or orthokeratology as it is currently known has steadily increased, providing the basis for a rebirth of this technique. ${ }^{1-4}$ Fitting contact lenses for overnight CRT generally begins with a short period of lens wear under open eye conditions until the optimal fit is achieved. ${ }^{5}$ Clinicians are aware that rapid changes happen even with short periods of lens trial, and more than 0.5 diopters (D) of apical corneal flattening have been observed after 10 minutes of reverse geometry lens wear under open eye conditions. ${ }^{1}$ The short-term response of the corneal tissue to CRT could be a reflection of the biomechanical response of the cornea to external forces or corneal rheology.

Rheology is the branch of science that deals with the flow and deformation of matter as a result of stress. This concept can be applied to several areas of biomedical science. Within the orthokeratology field, corneal rheology is of interest to estimate a predictable path for corneal deformation when corneal tissue is submitted to a given stress under a contact lens. ${ }^{6}$ Some recent investigations have evaluated the short-term response of the cornea to the effect of reverse geometry lenses at different time intervals under open and closed eye conditions. ${ }^{1,7,8}$ In addition, the reformation of the corneal tissue when the stress is relieved after lens removal is also of primary interest in 2 senses. At the scientific level, it is important to elucidate if changes that occur after lens application will recover at the same rate. These outcomes could provide additional understanding of the actual mechanism in regard to corneal shape changes with modern orthokeratology. From a clinical point of view, it is important to know what occurs to the corneal shape and refractive power of the cornea when the patient leaves the clinic after an orthokeratology fitting trial.

Some recent studies have investigated the recovery of the orthokeratologic effect in patients wearing reverse geometry contact lenses for myopia correction for several months after treatment discontinuation, ${ }^{9,10}$ and despite the fact that all patients had a full recovery, the time needed to do so varies among them. We have recently observed that the biomechanical properties of the cornea can influence the response and recovery of the orthokeratology effect in the short term, and this could explain, at least in part, the interindividual variation of the corneal response during CRT with reverse geometry contact lenses. ${ }^{11}$ To fully understand the rheology of the cornea under short-term wear of reverse geometry lenses, the progression and recovery changes in corneal shape were investigated to establish the pattern of onset and 
recovery of topographic changes induced by CRT lenses after short periods of lens wear and how this changes depending on the refractive target of the treatment. Eventually, the pathway of such changes could also provide further information regarding the mechanism behind CRT topographic and refractive changes.

With this aim, the present work has been designed to evaluate the short-term response of the corneal topography within a period of 3 hours wearing reverse geometry for 2 different refractive targets under open eye conditions and during an additional period of 3 hours after lens removal.

\section{MATERIALS AND METHODS}

Fourteen volunteers were fitted with Paragon CRT (Paragon Vision Sciences, Mesa, AZ) rigid gas permeable contact lenses according to the fitting recommendations of the manufacturer simulating a different treatment effect of -2.00 and -4.00 myopia correction in the right and left eyes in random order. More details about the fitting procedure including optimal fluorescein pattern, corneal coverage, and lens centration and movement and guidelines of parameter change to achieve optimal fitting are described in the literature. ${ }^{12}$ After the ideal fit was achieved on a trial experience (first visit), the patients returned at least 1 week later in the morning (second visit) and baseline measurements were obtained before lens insertion. To minimize the effects of physiologic edema and diurnal variations of corneal thickness $^{13}$ and corneal curvature ${ }^{14}$ (both can potentially affect corneal topographic stability) during the first hours after awaking, the patients were asked to wake up at least 3 hours before attending the clinic and all experiments were performed between $10 \mathrm{AM}$ and $4 \mathrm{PM}$. After the lenses were placed in both eyes, subsequent measurements were obtained at $30(30), 60$ (60), and 180 minutes (180); the same measurements were also taken at 30 (210), 60 (240), and 180 minutes (360) after lens removal. To minimize the effect of lens removal on subsequent orthokeratologic effect, the time of intermediate examinations was limited to a maximum of 5 minutes, the time required to perform topographic measurements.

Baseline measurements of corneal topography were obtained using Medmont E300 corneal topographer (Medmont Pty, Ltd, Melbourne, Australia). Subsequent topographic readings were obtained at 30,60 , and 180 minutes during lens wear and 30, 60, and 180 minutes after lens removal or 210, 240, and 360 minutes, respectively (Fig. 1 as an example of sequential topographic difference maps).

Because of the known influence of age on orthokeratology, ${ }^{8}$ the subjects' age was limited between 19 and 22 years $(20.3 \pm 3.2$ years). Other inclusion criteria also required that the subjects had no history of corneal surgery or active disease or corneal abnormality or had been previously under orthokeratology treatment. After data collection, 2 eyes in each refractive target group were not included in subsequent analysis. One eye was not included because of central punctuate keratopathy consisting of slight epithelial roughness with no signs of inflammation or infiltrates at one of the intermediate visits and another because of central island formation. Twelve eyes remained in each one of the refractive groups.
Data were analyzed using the statistical package, SPSS version 14.0., and Kolmogorov-Smirnov test was used to check normal distribution of variables. Changes from baseline were tested for statistical significance using $t$ test with Bonferroni post hoc correction. Comparison of the response between eyes with -2.00 and -4.00 treatments was established using 1-way analysis of variance. The level of significance was established at $\alpha=0.05$.

\section{RESULTS}

Changes from baseline at different time intervals for apical corneal curvature, keratometric data, and corneal shape are shown in Figures 2A-E, respectively. Table 1 presents F2|T1 baseline values and changes in corneal parameters along with their respective statistical significance. Baseline values were not significantly different between both refractive groups (analysis of variance, $P>0.05$ ). Significant changes are evident after 30 minutes of lens wear for most of the parameters. It is evident that the rate of change is much faster during the first 30 minutes for the majority of corneal parameters, particularly for corneal curvature values.

Changes in corneal parameters display a biphasic behavior during a lens wear period, showing an initial rapid onset of the orthokeratologic effect and a subsequent period of slower changes when the response between both treatments differentiates and the gap remains constant thereafter. No differences were observed between the -2.00 and -4.00 groups for most parameters during this initial period. Conversely, between the 30-minute and the 3-hour visits, differences in corneal response between both refractive groups become evident although not statistically significant, except for flat keratometry at 60 minutes after lens removal (240 minutes).

Recovery of the orthokeratologic effect after lens removal is almost as rapid as its onset in this short-term study of lens wear under open eye conditions. Although curvature changes follow an almost mirror behavior during the 3 hours of lens wear and the 3 hours after lens removal, changes in corneal shape do not respond in the same way. Overall, shape changes last longer than local (apical) or zonal (keratometry) curvature changes. After 3 hours, statistically significant differences from baseline still remain for flat keratometry and corneal eccentricity.

\section{DISCUSSION}

To our knowledge, this is the first study reporting the short-term effect and recovery response of the human cornea to sigmoid reverse geometry contact lenses under open eye conditions during 3 hours of orthokeratologic treatment and an additional 3-hour period of recovery. Present results show how the cornea can change its shape in response to orthokeratology and safely recover to the baseline values of curvature almost at the same rate after lens removal after a short-term period of lens wear under open eye conditions.

The changes found in the present study were similar to changes in apical radius in a similar study reported by Sridharan and Swarbrick ${ }^{1}$ but limited to the onset period. They 

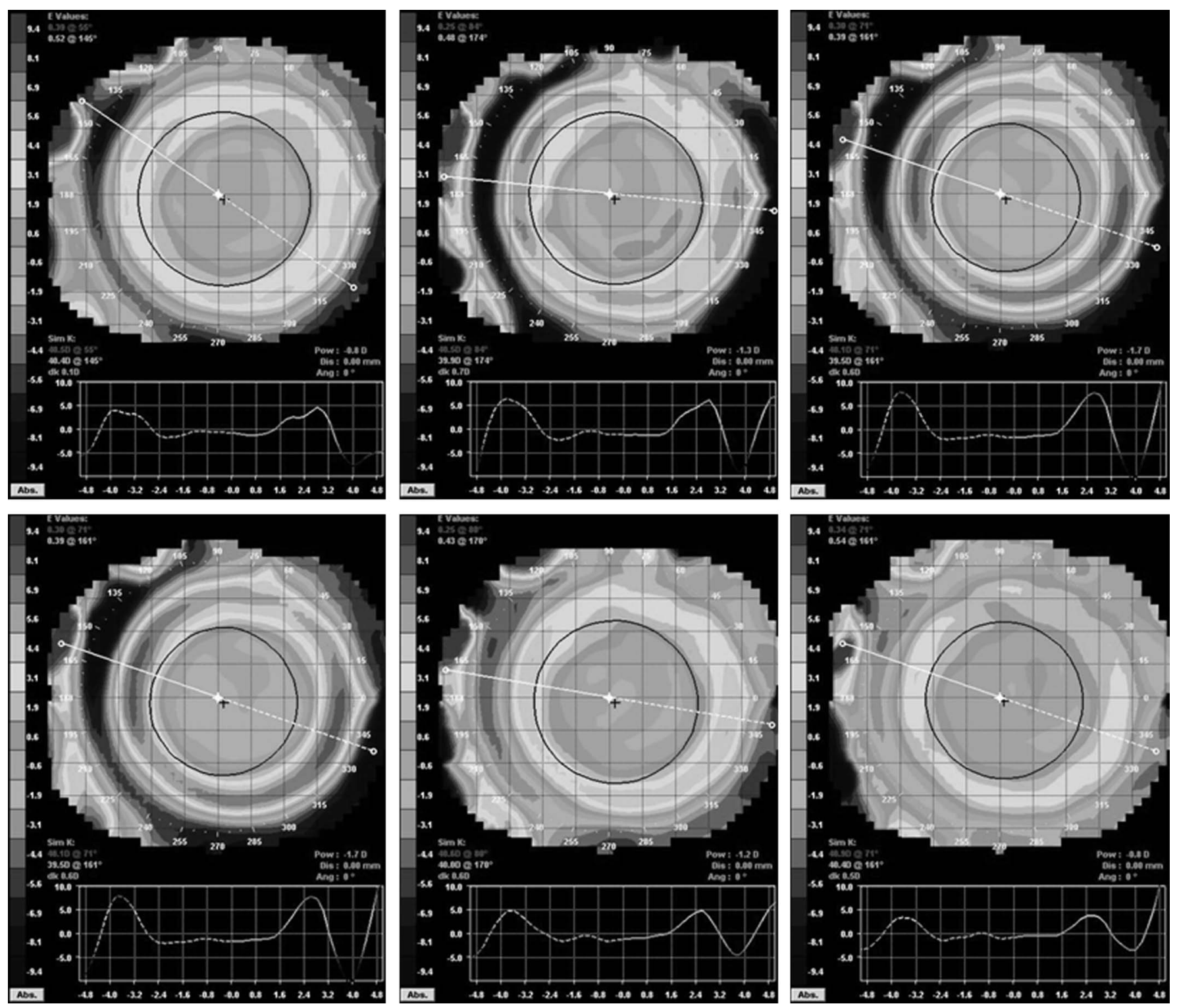

FIGURE 1. Time series of difference tangential curvature maps obtained with Medmont E300 for a typical patient within the sample of the study. Images represent differences between tangential curvature at 30, 60, and 180 minutes during progression (on top, left to right) and recovery phases (bottom, left to right) against baseline topography. As an example, apical curvature differed by 0.8 , 1.3 , and $1.7 \mathrm{D}$ from baseline at 30,60, and 180 minutes during lens wear and by $1.2,0.8$, and $0.2 \mathrm{D}$ at 30,60 , and 180 minutes, respectively, after lens removal for a $-4.00-\mathrm{D}$ attempted correction.

reported a change in apical power of $0.61 \mathrm{D}$ after 10 minutes of lens wear, similar to the change found by us at the 30-minute visit for -2.00 and -4.00 target refraction groups. Sridharan and Swarbrick also found a less change in keratometric power compared with apical power, and the more rapid changes occur in the first 10-30 minutes of lens wear. In their study, the additional change in corneal refractive power found after 60 minutes of lens wear was almost identical to the one observed within the first 10 minutes. Moreover, even after 8 hours of overnight wear, the rate of change is low compared with the first 10-30 minutes. Our results completely support this behavior of quick short-term changes (during the first 10-30 minutes) and slowdown pattern after this initial period with the additional benefit of follow-up during the recovery period. A recent report by $\mathrm{Lu}$ et $\mathrm{al}^{15}$ has also shown fast changes in response to hypermetropic orthokeratology after 15 minutes of lens wear.

Another recent study investigated the short-term changes in uncorrected visual acuity; spherical equivalent refraction; and central flat and steep keratometry after having worn Contex OK and Paragon CRT reverse geometry contact lenses under closed eye conditions for 60 minutes. Results displayed an average change of $0.50-0.75 \mathrm{D}$ of spherical equivalent, associated with a reduction on flat and steep 

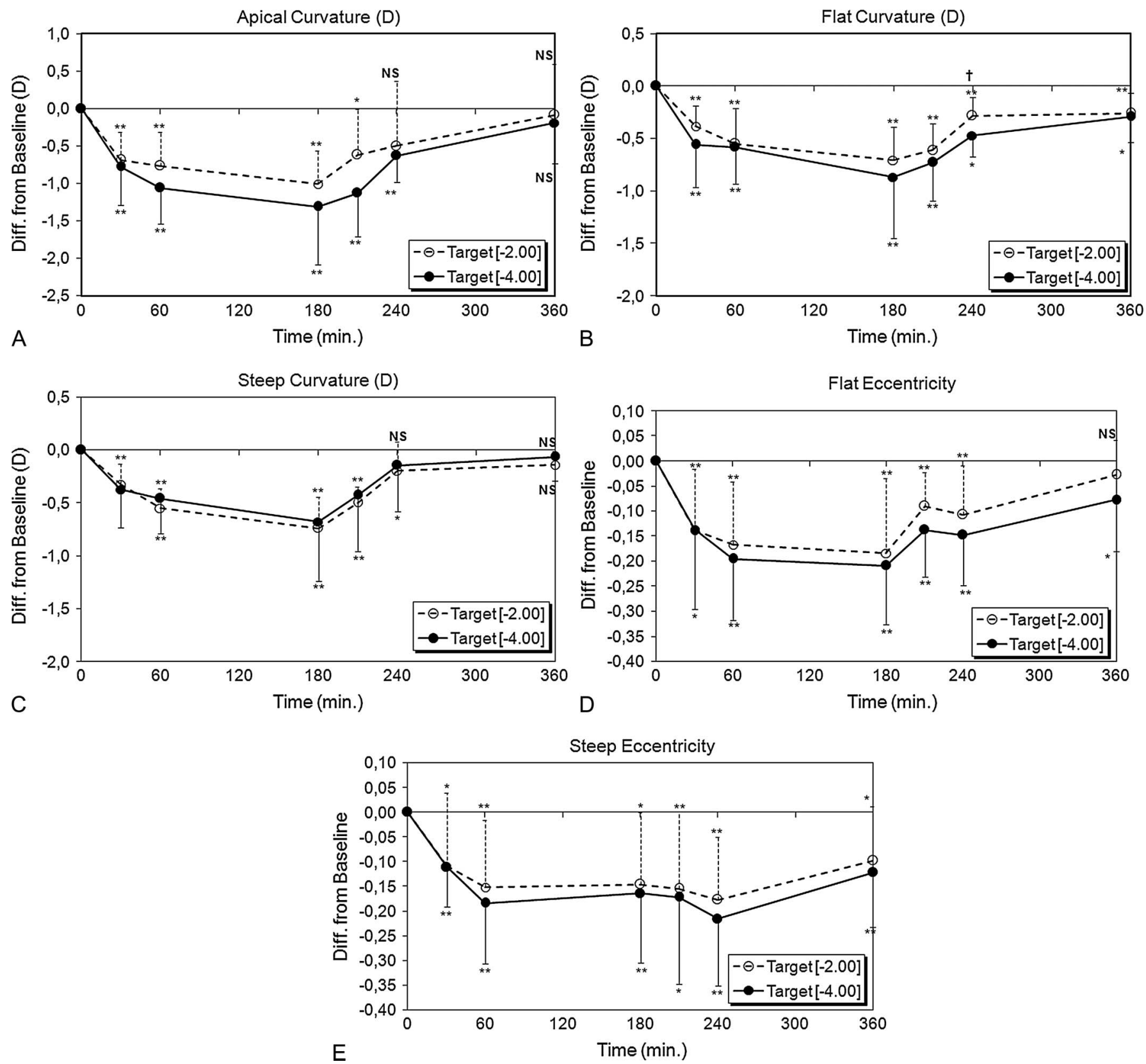

FIGURE 2. Changes in corneal parameters from baseline at different time intervals, with contact lenses on the eye ( 30,60 , and 180 minutes) and after contact lens removal (210, 240, and 360 minutes). Apical curvature (A), flat keratometric reading (B), steep keratometric reading (C), eccentricity in the flat meridian (D), and eccentricity in the steep meridian (E). Error bars indicate the SD. ${ }^{*} P<0.05$ for changes against baseline. $\dagger P<0.05$ for changes between refractive targets. NS, nonsignificant.

AU6 keratometry of about $0.5-0.7$ D. ${ }^{7}$ Their study was carried out under closed eye conditions, and our keratometric changes under open eye conditions agree with those findings.

Jackson et al have studied visual acuity, corneal topography, and corneal thickness after short periods of Paragon CRT lens wear. They fitted 20 eyes of 10 patients and measured unaided high-contrast visual acuity, apical corneal radius, and central corneal thickness after separated periods of 10, 30, and 60 minutes. Results showed a significant improvement in visual acuity after 30 minutes and significant changes in apical corneal radius after 10 minutes. However, they failed to detect significant corneal thickness changes with an ultrasound pachymetry. ${ }^{16} \mathrm{Lu}$ et al fitted Paragon CRT in 1 eye of 20 volunteers, whereas the contralateral eye wore a tetracurve RGP lens designed to be fitted in an alignment pattern. Topographic analysis was collected at baseline, after one night of lens wear, and again after the lens was discontinued for 12 hours. Topographic measurements were taken along the horizontal meridian, centrally and midperipherally at $3 \mathrm{~mm}$. Changes in corneal curvature revealed 
TABLE 1. Average Baseline Values and Changes in Corneal Parameters from Baseline at Different Time Intervals During Lens Wear and after Lens Removal

\begin{tabular}{|c|c|c|c|c|c|c|c|c|c|c|c|}
\hline & \multirow{3}{*}{$\begin{array}{c}\text { Parameter } \\
\text { Target Treatment } \\
\text { Baseline values }\end{array}$} & \multicolumn{2}{|c|}{ Apical Power } & \multicolumn{2}{|c|}{ Flat Sim'K } & \multicolumn{2}{|c|}{ Steep Sim'K } & \multicolumn{2}{|c|}{ Flat Eccentricity } & \multicolumn{2}{|c|}{ Steep Eccentricity } \\
\hline & & -2.00 & -4.00 & -2.00 & -4.00 & -2.00 & -4.00 & -2.00 & -4.00 & -2.00 & -4.00 \\
\hline & & $\begin{array}{c}43.17 \\
(1.53)\end{array}$ & $\begin{array}{l}42.99 \\
(1.31)\end{array}$ & $\begin{array}{c}42.75 \\
(1.31)\end{array}$ & $\begin{array}{c}42.65 \\
(1.46)\end{array}$ & $\begin{array}{c}43.65 \\
(1.43)\end{array}$ & $\begin{array}{c}43.52 \\
(1.45)\end{array}$ & $\begin{array}{c}0.66 \\
(0.13)\end{array}$ & $\begin{array}{c}0.63 \\
(0.12)\end{array}$ & $\begin{array}{c}0.43 \\
(0.10)\end{array}$ & $\begin{array}{c}0.42 \\
(0.10)\end{array}$ \\
\hline \multirow[t]{3}{*}{ Effect } & 30 & $\begin{array}{r}-0.68 * \\
(0.37)\end{array}$ & $\begin{array}{c}-0.77 * \\
(0.51)\end{array}$ & $\begin{array}{c}-0.38 * \\
(0.20)\end{array}$ & $\begin{array}{r}-0.56^{*} \\
(0.40)\end{array}$ & $\begin{array}{c}-0.32 * \\
(0.20)\end{array}$ & $\begin{array}{c}-0.37 * \\
(0.36)\end{array}$ & $\begin{array}{r}-0.14 * \\
(0.12)\end{array}$ & $\begin{array}{c}-0.14^{*} \\
(0.16)\end{array}$ & $\begin{array}{r}-0.11 * \\
(0.15)\end{array}$ & $\begin{array}{r}-0.11 * \\
(0.08)\end{array}$ \\
\hline & 60 & $\begin{array}{r}-0.76^{*} \\
(0.45)\end{array}$ & $\begin{array}{c}-1.06^{*} \\
(0.48)\end{array}$ & $\begin{array}{c}-0.55^{*} \\
(0.34)\end{array}$ & $\begin{array}{c}-0.58 * \\
(0.35)\end{array}$ & $\begin{array}{c}-0.55^{*} \\
(0.19)\end{array}$ & $\begin{array}{r}-0.46^{*} \\
(0.33)\end{array}$ & $\begin{array}{c}-0.17 * \\
(0.13)\end{array}$ & $\begin{array}{c}-0.20 * \\
(0.12)\end{array}$ & $\begin{array}{r}-0.15^{*} \\
(0.14)\end{array}$ & $\begin{array}{r}-0.18^{*} \\
(0.12)\end{array}$ \\
\hline & 180 & $\begin{array}{c}-1.01 * \\
(0.45)\end{array}$ & $\begin{array}{c}-1.31 * \\
(0.77)\end{array}$ & $\begin{array}{c}-0.71 * \\
(0.32)\end{array}$ & $\begin{array}{c}-0.87^{*} \\
(0.58)\end{array}$ & $\begin{array}{c}-0.74 * \\
(0.30)\end{array}$ & $\begin{array}{r}-0.68 \\
(0.56)\end{array}$ & $\begin{array}{c}-0.18^{*} \\
(0.15)\end{array}$ & $\begin{array}{c}-0.21 * \\
(0.12)\end{array}$ & $\begin{array}{r}-0.15^{*} \\
(0.15)\end{array}$ & $\begin{array}{r}-0.16^{*} \\
(0.14)\end{array}$ \\
\hline \multirow[t]{3}{*}{ Recovery } & 210 & $\begin{array}{c}-0.61 \\
(0.60)\end{array}$ & $\begin{array}{c}-1.13^{*} \\
(0.58)\end{array}$ & $\begin{array}{c}-0.61 * \\
(0.26)\end{array}$ & $\begin{array}{c}-0.73 * \\
(0.36)\end{array}$ & $\begin{array}{r}-0.50 * \\
(0.15)\end{array}$ & $\begin{array}{r}-0.43 \\
(0.53)\end{array}$ & $\begin{array}{c}-0.09 * \\
(0.07)\end{array}$ & $\begin{array}{c}-0.14^{*} \\
(0.09)\end{array}$ & $\begin{array}{r}-0.16^{*} \\
(0.16)\end{array}$ & $\begin{array}{c}-0.17^{*} \\
(0.18)\end{array}$ \\
\hline & 240 & $\begin{array}{r}-0.50 \\
(0.87)\end{array}$ & $\begin{array}{c}-0.63 * \\
(0.35)\end{array}$ & $\begin{array}{c}-0.28 * \\
(0.18)\end{array}$ & $\begin{array}{c}-0.47 * \\
(0.20)\end{array}$ & $\begin{array}{c}-0.20 \\
(0.28)\end{array}$ & $\begin{array}{r}-0.15 \\
(0.43)\end{array}$ & $\begin{array}{c}-0.11 * \\
(0.10)\end{array}$ & $\begin{array}{c}-0.15^{*} \\
(0.10)\end{array}$ & $\begin{array}{r}-0.18^{*} \\
(0.13)\end{array}$ & $\begin{array}{r}-0.22^{*} \\
(0.14)\end{array}$ \\
\hline & 360 & $\begin{array}{r}-0.08 \\
(0.68)\end{array}$ & $\begin{array}{c}-0.19 \\
(0.54)\end{array}$ & $\begin{array}{c}-0.26^{*} \\
(0.19)\end{array}$ & $\begin{array}{c}-0.29 * \\
(0.24)\end{array}$ & $\begin{array}{r}-0.14 \\
(0.13)\end{array}$ & $\begin{array}{r}-0.07 \\
(0.23)\end{array}$ & $\begin{array}{c}-0.03 \\
(0.07)\end{array}$ & $\begin{array}{c}-0.08 * \\
(0.10)\end{array}$ & $\begin{array}{r}-0.10^{*} \\
(0.11)\end{array}$ & $\begin{array}{r}-0.12 * \\
(0.11)\end{array}$ \\
\hline
\end{tabular}

Values in parentheses are SD. The $t$ test with Bonferroni post hoc.

$* P<0.05$

central corneal power of $1.49 \mathrm{D}$ less and an increase of $1.96 \mathrm{D}$ at periphery for the eyes wearing reverse geometry lenses, whereas the contralateral eyes wearing standard geometry lenses displayed a mean reduction of corneal curvature of 0.37 and 0.15 at center and midperipheral locations, respectively. ${ }^{17}$ Soni and Nguyen randomly fitted 19 patients with reverse geometry RGP contact lenses to be $1.00 \mathrm{D}$ flatter than the central horizontal keratometric reading. After 60 minutes, they found a significant central corneal flattening of $1.01 \mathrm{D}$, but no differences at $1.5 \mathrm{~mm}$ from center and $-0.95 \mathrm{D}$ steepening at $2.5 \mathrm{~mm}$ from center. Central change correlated significantly with an overall reduction on refractive error of $0.80 \mathrm{D}$ and a significant improvement in uncorrected visual acuity. ${ }^{18}$

In the present study, steepest keratometry and corneal eccentricity followed a similar pattern of quicker changes within the first 30 minutes followed by a slowdown period.

Swarbrick et $\mathrm{al}^{2}$ and Alharbi and Swarbrick ${ }^{19}$ have reported that in orthokeratology, the corneal flattening and subsequent reduction in myopia are related to a central epithelial thinning. Mountford ${ }^{20}$ has postulated that unequal tear film pressure beneath the corneal reshaping contact lens is the driving force responsible for the flattening of the central epithelium and steepens the midperipheral epithelium. Today, there are 2 possible mechanisms to explain these rapid epithelial changes: (1) epithelial cell redistribution and (2) epithelial cell compression. Considering the previous scientific evidence, it seems that such quick redistribution and recovery of the epithelial thickness would be possible as a result of epithelial cell compression rather than epithelial cell redistribution and this is in agreement with the observations of samples of excised corneas from cats. ${ }^{21}$

Differences between the progression of topographic flattening suggest that other factors in addition to corneal thinning could play a role to explain anterior corneal flattening in the short-term response to orthokeratology lenses. One possible mechanism could be the overall corneal bending during the first moments of reverse geometry lens wear for myopia correction. The results of Owens et $\mathrm{al}^{22}$ which confirmed short-term flattening of the posterior cornea during orthokeratology, support this hypothesis.

Comparing the response of both groups $(-2.00$ vs -4.00 target), we did not find statistically significant differences in corneal response in the majority of visits. This could be explained in part by the limited sample size and the relatively large variability of response within each group. Despite this, differences between refractive targets are clinically appreciable after the first phase (first 30 minutes). Conversely, the second phase showed more evident differences between groups. Changes in apical curvature and flat keratometric radius were greater in the -4.00 refractive group. There were no differences between groups for steep keratometric radius and flat or steep eccentricity. Recovery changes were greater in the -2.00 refractive group. The first 30 minutes was the time frame that presented greater changes in proportion to the shorter period, both during progression and recovery phases. Differences between refractive treatments could be more significant during overnight wear. Eiselt et $\mathrm{al}^{23}$ showed that changes in flat and steep keratometric readings are 1.5-2 times greater in patients above $-3.25 \mathrm{D}$ of myopia correction than in patients below $-3.00 \mathrm{D}$ of myopia correction.

During the recovery phase, we also observed that the -2.00 and -4.00 treatment groups returned to baseline at a different rate. Corneal eccentricity returned to baseline slower in the -4.00 group. Conversely, apical radius and flat keratometry returned to baseline faster for the -4.00 group, leading to an almost identical residual value between both groups 3 hours after lens removal. Three hours after lens removal, the flat keratometric value and eccentricity had not returned to baseline for both groups.

From the clinical point of view, the present results will help clinicians to know what to expect from the brief periods of lens wear under open eye conditions during lens trials and what to expect after the patient leaves their rooms. The onset of 
corneal changes is so rapid that even with short periods of lens wear, the cornea will suffer significant changes that will mask the effect of subsequent lens trials if a reasonable period has not elapsed before the insertion of the next lens. This does not mean that after a trial lens is applied, another cannot be applied because it is not expected that the changes that may have occurred will affect the fitting of the next lens. In clinical practice, it is usual to observe that the fitting of the lens does not change significantly even after a week of lens wear when most of the topographic changes had taken place. For example, if the first lens does not center well, a second lens could be placed on the eye to evaluate cornea-lens relationship. In this case, the clinician should be aware of the possible imprint of the first lens altering the effect of the second lens on the corneal topography. After the initial lens is applied and allowed to remain for 10-30 minutes, the response of the corneal shape will be evident. Conversely, when a second lens is applied, whatever effect detected would be influenced by the first trial lens. Therefore, we suggest the fitte to evaluate the lens within 5 minutes on lens wear.

In summary, we have reported interesting findings regarding the ability of the corneal tissue to respond to CRT in the short term for different refractive targets. Short-term changes in apical radius in response to CRT lens wear occur within 30 minutes. In addition, apical power and steepest keratometric power return no baseline after short periods of wear ( 3 hours). Differentiation of corneal response between different refractive targets occurs after an initial period or about 30 minutes, characterized by a uniform corneal response irrespective of the attempted refraction to be corrected. This work shows that the response of the cornea to CRT in the short term could be very important to elucidate the biomechanics of the cornea in response to CRT. Interesting results correlating the biomechanical properties of the cornea with the response to CRT have been published recently by the authors. ${ }^{11}$ This issue is a key factor to better understand the corneal response to orthokeratology and to better predict the outcomes. From the clinical point of view, this work helps us to understand what to expect from the cornea while we are performing the fitting tests and what to expect after the patient leaves the clinic. Nevertheless, the most important result from this study is the confirmation that the cornea can easily deform and recover its original shape after short periods of CRT wear for corneal reshaping.

\section{ACKNOWLEDGMENTS}

The authors wish to thankInterlenco S.A. for the loan of the Paragon CRT Diagnostic and Dispensing System. C.V.C. and J.M.G.-M. are onthe Interlenco speakers bureau, otherwise, none of the authors have a commercial or financial interest in the instruments or materials used in the study. The authors wish to thank the assistance of Dr. Filomena Louro from the program of Support to the Edition of Scientific Papers at the University of Minho for help with English editing of the article.

\section{REFERENCES}

1. Sridharan R, Swarbrick H. Corneal response to short-term orthokeratology lens wear. Optom Vis Sci. 2003;80:200-206.

2. Swarbrick HA, Wong G, O'Leary DJ. Corneal response to orthokeratology. Optom Vis Sci. 1998;75:791-799.

3. Swarbrick HA. Orthokeratology review and update. Clin Exp Optom. 2006;89:124-143.

4. Mountford J. History and general principles. In: Mountford J, Ruston D, Dave T, eds. Orthokeratology. Principles and Practice. Oxford, United Kingdom: Butterworth-Heinemann; 2004:1-16.

5. Villa-Collar C, Gonzalez-Meijome JM. Proceso de adaptación en ortoqueratologia nocturna. In: Villa-Collar C, Gonzalez-Meijome JM, eds. Ortoqueratologia Nocturna. Madrid, Spain: ICM; 2006:97-147.

6. Mountford J. Orthokeratology. Contact Lenses. London, United Kingdom: Butterworth-Heinemann; 1997:653-692.

7. Kamei Y, Cassar K, Shen J, et al. Short-term corneal changes in closed eye condition with orthokeratology lenses. ARVO meeting abstracts. 2005; 46:2060.

8. Jayakumar J, Swarbrick HA. The effect of age on short-term orthokeratology. Optom Vis Sci. 2005;82:505-511.

9. Barr JT, Rah MJ, Meyers W, et al. Recovery of refractive error after corneal refractive therapy. Eye Contact Lens. 2004;30:247-251.

10. Soni PS, Nguyen TT, Bonanno JA. Overnight orthokeratology: refractive and corneal recovery after discontinuation of reverse-geometry lenses. Eye Contact Lens. 2004;30:254-262.

11. Gonzalez-Meijome J, villa-collar C, Queiros A, et al. A pilot study on the influence of corneal biomechanical properties over the short term in response to corneal refractive therapy for myopia. Cornea. In press.

12. Gonzalez-Meijome JM, Villa-Collar C. Nomogram, corneal topography, and final prescription relations for corneal refractive therapy. Optom Vis Sci. 2007;84:59-64.

13. Du TR, Vega JA, Fonn D, et al. Diurnal variation of corneal sensitivity and thickness. Cornea. 2003;22:205-209.

14. Handa T, Mukuno K, Niida T, et al. Diurnal variation of human corneal curvature in young adults. $J$ Refract Surg. 2002;18:58-62.

15. Lu F, Simpson T, Sorbara L, et al. Malleability of the ocular surface in response to mechanical stress induced by orthokeratology contact lenses. Cornea. 2008;27:133-141

16. Jackson JM, Bildstein T, Anderson J, et al. Short-term corneal changes with corneal refractive therapy (CRT). Invest Ophthalmol Vis Sci. 2004; 45:1547.

17. Lu FH, Sorbara L, Kort RA, et al. Topographic keratometric effects of corneal refractive therapy after one night of lens wear. Invest Ophthalmol Vis Sci. 2003;44:3699.

18. Soni P, Nguyen T. Which corneal parameter, anterior corneal curvature, posterior corneal curvature, or corneal thickness is most sensitive to acute changes with reverse geometry orthokeratology lenses. Invest Ophthalmol Vis Sci. 2002;43:3086.

19. Alharbi A, Swarbrick HA. The effects of overnight orthokeratology lens wear on corneal thickness. Invest Ophthalmol Vis Sci. 2003;44: $2518-2523$.

20. Mountford J. A model of forces acting in orthokeratology. In: Mountford J, Ruston D, Dave T, eds. Orthokeratology. Principles and Practice. Oxford, United Kingdom: Butterworth-Heinemann; 2004: 269-301.

21. Choo JD, Caroline PJ, Harlin DD, et al. Morphologic changes in cat epithelium following continuous wear of orthokeratology lenses: a pilot study. Cont Lens Anterior Eye. 2008;31:29-37.

22. Owens H, Garner LF, Craig JP, et al. Posterior corneal changes with orthokeratology. Optom Vis Sci. 2004;81:421-426.

23. Eiselt T, Horton D, Nguyen TT, et al. Rate of change in corneal curvature, refractive error and visual acuity with Paragon CRT lenses. Optom Vis Sci. 2004;81:71. 